available for SSCN synthesis; however, each has its own set of drawbacks. One synthetic technique, the Stöber sol-gel method, leads to uniform silica-coated nanospheres but it suffers from low loading of magnetic material and requires an extra surface functionalization step. With aerosol pyrolysis, another approach, combustion of iron and silica precursors forms high-loading composites but the process requires high temperatures that can lead to nanosphere aggregation. In another technique, reverse microemulsion, magnetic material loading is determined by the amount of hydrophilic iron oxide dispersed in water droplets that are suspended in a continuous oil phase. Highquality hydrophilic iron oxide particles are an important part of this technique, but their synthesis is still a challenge. The oil-in-DEG microemulsion technique described in this report uses high-quality hydrophobic iron oxide, does not require high temperatures, and is capable of high magnetic material loading.

In the described microemulsion technique, hydrophobic iron oxide nanoparticles are dispersed in a discrete tetraethylorthosilicate (TEOS) phase. The magnetic material-containing oil droplets are stabilized in a DEG continuous phase using a nonionic surfactant. The use of DEG instead of water eliminates premature hydrolysis and condensation of the TEOS giving the researchers greater control over particle size and agglomeration. Ammonia is added which diffuses into the oil droplets causing the hydrolysis and condensation reactions to occur only in the oil droplets. This eliminates the possibility of empty SSCNs. As a result, the researchers have made SSCNs with saturation magnetizations as high as $33.6 \mathrm{emu} / \mathrm{g}$ (compared to pure superparamagnetic iron oxide nanoparticles with a saturation magnetization of $\sim 42 \mathrm{emu} / \mathrm{g}$ ), much higher than reverse microemulsion methods that typically provide less than $5 \mathrm{emu} / \mathrm{g}$. In the search for highly magnetic silica-coated particles, the research group has introduced a synthetic method that holds great potential for improving future biological and environmental separations.

KEVIN HERLIHY

\section{Novel Hydrogel Membrane Enables Demonstration of Water Transpiration in a Synthetic Tree}

Transpiration-the motion of water from the soil into the air through a vascular plant-involves differences in pressure about a hundred times larger than those currently achieved in synthetic wicks. T.D. Wheeler and A.D. Stroock from Cornell University recently overcame this chal- lenge by designing and operating a synthetic tree, a microfluidic system formed in a cross-linked organic hydrogel.

As the researchers reported in the September 11 issue of Nature (DOI:10.138/ nature07226; p. 208), the system captures the main attributes of transpiration in plants: transduction of subsaturation in the vapor phase of water into negative pressures in the liquid phase, stabilization and flow of liquid water at large negative pressures (down to $-21 \mathrm{MPa}$ ), continuous heat transfer with the evaporation of liquid water at negative pressure, and continuous extraction of liquid water from subsaturated sources.

The researchers first created 1-mm thick sheets of poly(hydroxyethyl methacrylate) (pHEMA); a sheet was imprinted with microfluidic channels by soft lithography, and then another sheet was bonded on top of it by repolymerization. The system contained all the elements required by a synthetic tree: a root membrane of pHEMA in contact with the water source, the liquidfilled xylem capillary, and a leaf membrane of pHEMA exposed to air.

A steady-state flow through the synthetic tree was achieved by exposing the leaf to air flow. The root was exposed either to vapor or liquid water, and it was demonstrated that the leaf network can act alone as a pump due to the highly negative pressure differences. Pressures 15-fold higher than any previously reported synthetic pumped system were recorded when the root membrane was punctured for easier access to the source, and separate experiments with an intact root membrane demonstrated the capacity of the system to reproduce the transpiration of water in a plant.

Through a series of experiments, the researchers demonstrated that the system offers a platform not only for realistic investigations of different aspects of plant physiology, but also for applications in processes demanding large pressure differences (that can move liquid tens of meters above a reservoir), in cooling a heat source by transpiration, a mechanism that will greatly increase the heat flux compared to other technologies, and in the extraction and purification of water from subsaturated soils.

EugEN PANAITESCU

\section{Hydrogels Stimulated by Antibiotics to Release Vascular Endothelial Growth Factors}

Polymeric hydrogels can be triggered to release pharmacological agents by chemical, biological, and physical means. However, a significant therapeutic level is difficult to achieve because the stimulus concentration required to elicit a polymer response cannot easily be applied in vivo. A hydrogel based on pharmacologic processes has been designed and validated by M. Ehrbar of the University Hospital Zurich, R. Schoenmakers and W. Weber of ETH Zurich, and their collaborators to release the protein vascular endothelial growth factor (VEGF) upon interaction with the antibiotic Novobiocin.

As described in the October 2008 issue of Nature Materials (DOI: 10.1038/nmat2250; p. 800), the researchers designed an antibiotic-sensing hydrogel by grafting bacterial gyrase subunit B (GyrB) onto polyacrylamide, then dimerizing GyrB with the antibiotic Coumermycin to stabilize the structure. The hydrogel was designed to release its contents when it came in contact with a secondary antibiotic, Novobiocin. Novobiocin binds to the GyrB and competitively inhibits the GyrB-Coumermycin interaction. Once GyrB is uncoupled, the hydrogel structure is loosened and dissolution occurs. To validate the feasibility of the hydrogel, swelling behavior and cell activity was examined.

The release of protein from prepared hydrogels was determined by incubating the hydrogels in a phosphate buffered saline (PBS) solution containing concentrations of Novobiocin, ranging from $0 \mathrm{mM}$ to $1 \mathrm{mM}$. The hydrogels were monitored for a period of $120 \mathrm{~h}$, and the release profile showed that as concentrations of Novobiocin increased, the amount of protein released also increased. This effect was due to the rapid dissolution of the hydrogel in the presence of higher concentrations of Novobiocin, and slower release at lower concentrations. A cell proliferation assay, using human embryonic kidney cells, was used to study possible cytotoxic effects of the hydrogel components. The assay showed that no cytotoxic effects were present at hydrogel quantities sufficient to release VEGF concentrations of $>500 \mathrm{ng} \mathrm{ml}^{-1}$. To determine the bioavailability and bioactivity of VEGF after release from the hydrogel, a cell proliferation assay was performed using human umbilical vein endothelial cells (HUVEC). After incubating the hydrogels in the cell medium with and without Novobiocin for $96 \mathrm{~h}$, the assay showed in the absence of Novobiocin that there is no difference in cell proliferation compared to the negative control. However, when Novobiocin is present, there is a marked increase of HUVEC growth.

The novel stimuli-responsive hydrogel in this study has demonstrated the ability to release therapeutic treatments within physiological limits, without sacrificing the activity of its contents.

TARA D. WASHINGTON 